\title{
Rho GTPase Function in Flies: Insights from a Developmental and Organismal Perspective
}

\author{
James E. Johndrow ${ }^{1}$, Craig R. Magie ${ }^{1,2}$ and Susan M. Parkhurst ${ }^{1,2, *}$
}
${ }^{1}$ Division of Basic Sciences, Fred Hutchinson Cancer Research Center, Seattle, WA 98109, USA, and
${ }^{2}$ Department of Biology, University of Washington, Seattle, WA 98195, USA

\author{
Division of Basic Sciences; A1-162 \\ Fred Hutchinson Cancer Research Center \\ 1100 Fairview Avenue North \\ P.O. Box 19024 \\ Seattle, WA 98109-1024 \\ Tel: (206) 667-6466 \\ Fax: (206) 667-6497 \\ Email: susanp@fhcrc.org
}

Key words: Rho GTPases, Drosophila, development, effectors, loss-of-function mutation, dominant-negative, constitutively-active

* Author for correspondence (e-mail: susanp@fhcrc.org) 


\begin{abstract}
Morphogenesis is a key event in the development of a multicellular organism, and is reliant on coordinated transcriptional and signal transduction events. In order to establish the segmented body plan that underlies much of metazoan development, individual cells and groups of cells must respond to exogenous signals with complex movements and shape changes. One class of proteins that plays a pivotal role in the interpretation of extracellular cues into cellular behavior is the Rho family of small GTPases. These molecular switches are essential components of a growing number of signaling pathways, many of which regulate actin cytoskeletal remodeling. Much of our understanding of Rho biology has come from work done in cell culture. More recently, the fruit fly Drosophila melanogaster has emerged as an excellent genetic system for the study of these proteins in a developmental and organismal context. Studies in flies have greatly enhanced our understanding of pathways involving Rho GTPases and their roles in development.
\end{abstract}

\title{
Background
}

Rho family GTPases are found in a wide variety of eukaryotic species and are well conserved throughout evolution, consistent with their importance in the regulation of developmental processes (Burridge and Wennerberg, 2004; Etienne-Manneville and Hall, 2002; Settleman, 1999). The founding members of this family are Rho, Rac and Cdc42 (Hall, 1998; Mackay and Hall, 1998). Since the initial characterization of these proteins, a number of less conserved family members have also been identified including TC10 and the Rnd proteins in mammalian systems (Sahai and Marshall, 2002), and the Rop and Arac proteins in plants (Valster et al., 2000).

Rho proteins function as molecular switches, cycling between an active, GTP-bound state and an inactive, GDP-bound state. Their activation state is modulated by their intrinsic GTPase activity and the action of associated regulatory enzymes of three major classes: the guanine nucleotide exchange factors (GEFs), GTPase-activating proteins (GAPs), and GDP dissociation inhibitors (GDIs) (Fig. 1; (Maruta, 1998). GEFs activate Rho proteins by promoting the exchange of GDP for GTP. GAPs promote inactivation of Rho by increasing the rate of GTP hydrolysis. GDIs bind Rho-GDP in the cytoplasm and prevent nucleotide exchange, promoting the inactive state. In the prevailing model for Rho activation, extracellular cues initiate signal transduction pathways involving a number of different cell surface receptors, leading to activation of a particular GEF and the subsequent activation of Rho (Fig. 1; reviewed in (Kjoller and Hall, 1999). A large number of RhoGEFs have been identified to date. Some of these act specifically on a particular Rho family member, while others can act on multiple members (Hornstein et al., 2004). This specificity is likely important in determining the cellular response to activation of Rho family members.

The mammalian Rho family includes three homologues of Rho (RhoA, B, and C), four Rac homologues (Rac1, 2, and 3, and RhoG), and five Cdc42-like genes (Cdc42, TC10, TCL, Wrch1, and $\mathrm{Chp} / \mathrm{Wrch}$; Burridge and Wennerberg, 2004). Since it was initially thought that these proteins might act redundantly within each subfamily, mouse knockouts were not made. Fortunately, the nature of Rho GTPases as molecular switches allows for the construction of dominant-negative (DN) and constitutively-active (CA) versions of these proteins that are unable to release GDP or hydrolyze GTP, respectively. Overexpression of these mutant proteins, 
primarily in tissue culture experiments, led to the identification of roles for these proteins in a wide variety of cellular functions, including transcriptional activation, cell cycle progression, cell-cell and cell-substrate adhesion, secretion, endocytosis, phospholipid turnover, cytokinesis, and apoptosis (Braga, 1999; Coleman and Olson, 2002; Ellis and Mellor, 2000; Fukata and Kaibuchi, 2001; Hall, 1998; Mackay and Hall, 1998). This diversity of functions suggests that Rho proteins operate through multiple pathways in different contexts. A number of effector molecules have been identified that act downstream of Rho activation (Table 1; reviewed in (Bishop and Hall, 2000), though the mechanisms underlying the specificity of Rho action are incompletely understood. Notably, many of the known effectors of Rho family GTPases regulate actin dynamics and cytoskeletal architecture directly or through the activation of targets farther downstream. Thus, although it was originally assumed that Rho proteins would function analogously to their homologue Ras (by activating a kinase cascade that leads to transcriptional regulation), most of the pathways downstream of Rho family proteins converge on regulators of cell shape and migration. With few exceptions, transcriptional regulation associated with activation of Rho-mediated pathways is proving to be the indirect result of changes in cytoskeletal architecture. This point is well-illustrated by studies in Drosophila, where most of the phenotypes associated with loss of Rho GTPase activity are consistent with improper cell morphology, movement, or abnormalities in microfilament architecture (cf. Magie et al. 1999, 2002; Genova et al., 2000; Hakeda-Suzuki et al., 2002).

Rho, Rac, and $\mathrm{Cdc} 42$ were initially identified as regulators of the actin cytoskeleton. Injection of fibroblasts with activated forms of Rho, Rac, or Cdc42 results in responses characteristic of each of these proteins: Rho causes the formation of stress fibers (Ridley and Hall, 1992), Rac induces lamellipodia/membrane ruffling (Ridley et al., 1992), and Cdc42 triggers filopodia formation (Kozma et al., 1995; Nobes and Hall, 1995). The interdependence of different pathways involving Rho GTPases is somewhat unclear. For instance, a hierarchical relationship has been described in fibroblasts in which Cdc42 activates Rac, which in turn activates Rho (Nobes and Hall, 1995). In other cell types, however, this relationship has not been observed, and the effects Rho, Rac and Cdc42 have on actin architecture appear to be not only qualitatively different, but in some cases functionally opposite. For example, in neuronal precursors Rac and Cdc42 are involved in outgrowth of neurite extensions, whereas Rho is involved in their retraction (Kozma et al., 1997).

Initial work investigating Rho function was mainly done in tissue culture, utilizing overexpression of DN or CA versions of Rho proteins or bacterial toxins that inhibit Rho GTPases, such as C3 transferase (C3), specific for Rho, and Toxin B, which inhibits Rho, Rac, and Cdc42 (Boquet, 1999). In this context, the dominant negative was considered equivalent to loss-of-function. While a great deal has been learned from these studies regarding the biochemical nature of Rho signaling, studies using pharmacological inhibition or ectopic expression of dominantly acting mutants are vulnerable to nonspecific effects. In addition, they leave open the question of how Rho functions in a heterogeneous multicellular context. Genetic models, including Drosophila, have more recently emerged as potent systems in which to study Rho GTPase function in a developmental and organismal context.

Seven Rho family members have been identified in Drosophila: one Rho gene (Rhol), three Rac genes ( $R a c 1, R a c 2$ and $M t l$ ) and one $\mathrm{Cdc} 42$ gene ( $C d c 42)$, along with two other Rho family members, RhoL and RhoBTB, that are equally similar to Rho, Rac, and Cdc42. In cases where mammalian homologues can be assigned, fly Rho GTPases are $86 \%-92 \%$ identical to their mammalian counterparts. Initial investigation of Rho proteins during Drosophila development 
involved ectopic expression of DN or CA forms of these proteins. These studies linked Rho GTPases to a variety of morphogenetic processes in Drosophila oogenesis and embryogenesis (see Table 2; Glise et al., 1995; Harden et al., 1996; Harden et al., 1995; Murphy and Montell, 1996; Noselli, 1998; Riesgo-Escovar et al., 1996).

\section{Studies done with constitutively-active or dominant-negative alleles}

\section{Oogenesis}

Drosophila oogenesis has provided a valuable context in which to study the function of Rho GTPases in morphogenetic processes, as cell migration, cell shape changes, and cell-cell adhesion are all important to proper development of the oocyte. The Drosophila oocyte is contained within the egg chamber, a larger multicellular compartment that consists of both germline and somatic cells (Fig. 2A,B). The germline cells, which include the oocyte and its fifteen supporting nurse cells, are interconnected by actin-rich ring canals. These latter structures are the products of incomplete cytokinesis between the germ cells (Fig. 2B'-2B'), and allow for transport of maternal factors into the oocyte. During late oogenesis, the nurse cells dump their contents rapidly into the oocyte, a process that is dependent on contraction of the actin cytoskeleton. A population of somatic follicle cells surrounds the egg chamber. These cells undergo a series of migrations and cell shape changes during oogenesis. Border cells, a small population of follicle cells originating at the anterior pole of the egg chamber, undergo a remarkable migration at mid oogenesis by delaminating from their neighbors and crawling between the nurse cells to position themselves at the anterior border of the oocyte (Fig. 2A,B).

Expression of DN-Rac or DN-Cdc42 during oogenesis results in defects in the actin cytoskeleton (Murphy and Montell, 1996). DN-Rac inhibits border cell migration, presumably because these cells fail to produce the actin-rich protrusions that are seen during migration in wildtype egg chambers. Expression of CA- or DN- Cdc42 results in detachment of ring canals from the cell membrane and breakdown of the actin cytoskeleton, ultimately leading to nurse cell fusion, but neither DN- nor CA- Cdc42 appears to affect the follicle cells. When DN- forms of either of these GTPases are expressed later in oogenesis, failure of nurse cell dumping occurs because the nuclei are not properly anchored to the actin cytoskeleton and become trapped in the ring canals (Murphy and Montell, 1996). Thus, both Rac and Cdc42 are required for specific events associated with actin reorganization during oogenesis, with Rac being particularly important in the somatic follicle cells and Cdc42 necessary in the germline but apparently dispensable in the surrounding soma.

\section{Early Embryogenesis}

Early studies using DN-Rho GTPases also indicated a role for Rho1 in the early Drosophila embryo. The initial stages of embryonic development occur in a syncytium, in which nuclei divide within a common cytoplasm (Fig. 2C). The somatic nuclei divide synchronously for fourteen replicative cycles, at which point cellularization occurs. Cellularization can be considered a specialized form of cytokinesis, and like the cytokinetic contractile ring, the cleavage furrows that form to separate the blastoderm nuclei during cellularization accumulate high levels of F-actin and myosin-II. Cellularization involves the growth and extension of plasma membrane between the nuclei toward the interior of the embryo, resulting in a monolayer of cells termed the "cellular blastoderm" (Fig. 2D; reviewed in (Mazumdar and Mazumdar, 2002). Rho has been shown to be important for cytokinesis in systems such as early Xenopus 
and echinoderm embryos, as well as mammalian tissue culture cells (Kishi et al., 1993; Mabuchi et al., 1993). Not surprisingly, given the similarities between cytokinesis and Drosophila cellularization, injection of Drosophila embryos with C3 exoenzyme (Rho inhibitor) results in cellularization defects and severe disruption of actin organization, as does the injection of DNRho protein (Crawford et al., 1998).

Following cellularization, mitosis is no longer synchronous. Rather, a series of mitoses occur at stereotypical times and locations (termed "mitotic domains") throughout the later stages of embryogenesis (Foe, 1989). Drosophila Rhol also plays a role in mitosis through its effects on cytokinesis, mediated by the Rho-specific GEF encoded by pebble. Mutants lacking Pebble function fail to form a proper contractile ring at the cleavage furrow leading to an accumulation of multinucleate cells in regions undergoing mitosis (Prokopenko et al., 1999). Expression of DN-Rho1 results in a similar cytokinesis defect (Prokopenko et al., 1999), indicating that Rho1 is a primary regulator of actin-mediated contractility in cytokinesis and related events in early embryogenesis.

\section{Gastrulation}

Rho proteins in Drosophila are perhaps best characterized as regulators of the complex morphogenetic events that occur during gastrulation (Fig. 2E). Gastrulation begins when the ventral surface of the embryo flattens and continues with the cells in this region constricting their apical surfaces. These cells then invaginate into the interior of the embryo to form the ventral furrow, resulting in internalization of the early mesoderm. This is followed by two morphogenetic events in which the cells of the epidermis first migrate by intercalation along the dorsal surface of the embryo (germ band extension; Fig. 2Eiii-iv) and then appear to move ventrally in what is actually a series of cell shape changes (germ band retraction; Fig. 2Ev). This second event leaves a large hole in the dorsal epidermis, which is covered by extraembryonic amnioserosa cells (Fig. 2Evi). The hole is then closed by migration of the lateral epidermal sheet over the amnioserosa in a process known as dorsal closure (DC; Fig. 2Evii). This process requires the formation of an actin cable and extension of lamellipodia and filopodia by the dorsalmost row of epithelial cells, or leading edge (LE) cells (Jacinto et al., 2000). Lammelipodia are thought to be important for the migration of the LE cells over the amnioserosa. Interdigitation of filopodia at the conclusion of dorsal closure is required to zip the hole closed, and may play an important role in segmental matching with the cells of the opposing LE (Jacinto et al., 2000).

Rho GTPases are required throughout gastrulation. Expression of DN-Rho1 results in a delay in ventral furrow formation and the failure of some mesodermal precursors to be internalized properly, whereas DN-Rac or DN-Cdc42 do not have this effect, implicating Rhol as the primary regulator of the localized remodeling of the actin cytoskeleton necessary to cause the cell-shape changes that initiate gastrulation. Expression of DN- Rho, Rac, or Cdc42 all result in the failure to complete DC and a "dorsal open" phenotype characterized by holes in the dorsal cuticle (cf. Fig. 3C; Harden et al., 1999). Activation of the Jun N-terminal kinase (JNK) pathway is required for maintenance of the elongated morphology of LE cells and migration of the epithelial sheet over the amnioserosa (Glise et al., 1995; Kockel et al., 1997). Since expression of DN- Rho GTPases results in defects in both of these processes, Rho proteins have been hypothesized to act upstream of the JNK pathway during DC. This is consistent with studies in cell culture that link Rho GTPases to JNK pathway activation (Coso et al., 1995; Minden et al., 1995). In particular, there is some evidence that Rac and Cdc42 can activate JNK signaling through the binding of 
PAK kinases (Bagrodia et al., 1995; and see below). Expression of CA-Rac1 or CA-Cdc42 during DC results in ectopic activation of JNK signaling (Glise and Noselli, 1997).

Rho1 is required to properly organize the actomyosin cable at the LE, as expression of DNRho1 in a subpopulation of cells at the leading edge disrupts its formation in a cell autonomous manner (Jacinto et al., 2002). The cells lacking a cable due to expression of DN-Rho1 acquire hyperactive lamellipodial and filopodial protrusions and a migratory advantage over neighboring, wildtype cells, resulting in their overrepresentation at the dorsal midline. Expression of DN-Cdc42 in LE cells prevents the extension of filopodia from these cells, and adhesion of the opposing epithelium fails (Jacinto et al., 2000). The expression of DN-Cdc42 also results in mismatched segments, suggesting that filopodia are required for proper segment alignment.

Head involution, a process required for the formation of larval head structures and the internalization of the anterior portion of the embryo, occurs concomitantly with DC. Interestingly, while head involution clearly requires complex cell movements and shape changes, most mutants identified that specifically affect this process are regulators of apoptosis, as a stereotypical pattern of programmed cell death is necessary to allow head involution to occur normally (Nassif et al., 1998). In particular, mutations in the pro-apoptotic genes grim, reaper, and head involution defective (hid) all result in failure of head involution (Lee and Baehrecke, 2000). Involvement of Rho GTPases in head involution has not been specifically investigated in studies utilizing DN- or CA- constructs, but embryos zygotically null for Rhol fail to undergo head involution (discussed below).

\section{Other developmental processes}

Rho GTPases have also been linked to other developmental processes, most notably neuronal morphogenesis and the planar cell polarity (PCP) pathway. In the development of the nervous system, regulation of neuronal morphology is crucial to establishing proper synaptic contacts. Rho GTPases have been shown to mediate these cell-shape changes. Expression of either DNRac1 or CA-Rac1 in Drosophila causes abnormal filamentous actin accumulation and defects in axon outgrowth in peripheral neurons, while dendrites are not affected (Luo et al., 1994). Defects in axon morphology are also seen with expression of DN-Cdc42.

Planar cell polarity (PCP) is a developmental event common to many organisms through which polarized epithelial cells orient relative to one another and thereby acquire an additional axis of polarity in the plane of the epithelium. In Drosophila, examples of PCP include the orientation of hairs on the adult wing and of the ommatidia in the adult eye (Adler, 2002). In the wing, each cell orients itself proximal to distal, with an actin-based hair located at the distal edge. Whereas in the wing, single cells are independently involved in forming the overall polarity of the tissue, in the eye each group of cells comprising an ommatidium adopts polarity as a unit. In both cases, however, the genes involved in controlling the process are similar (reviewed in Mlodzik, 2002). Overexpression of DN-Cdc42 in the developing wing results in the failure of the distal hairs to form normally, and expression of DN-Rac1 at the same time and location indicates a role for Rac in specifying the position of the hair (Eaton et al., 1996). Expression of DN-Rac1 or CA-Rac1 in the eye also results in polarity defects, with Rac activity in this context required downstream of the Frizzled receptor (Fanto et al., 2000). Similarly, overexpression of Rho1 in the eye results in polarity defects (Hariharan et al., 1995), and CARho1 expression can rescue the Dishevelled PCP phenotype (Strutt et al., 1997). Taken together, 
these studies utilizing expression of DN- and/or CA- transgenes suggest pan-developmental roles for Rho GTPases in morphogenesis.

\section{Loss-of-function phenotypes for the Rho GTPases in Drosophila}

The studies discussed above suggest that, consistent with expression of DN- and CA- Rho GTPases in cell culture, Rho GTPases in Drosophila regulate morphogenesis through remodeling of the cytoskeleton. These studies support two major generalizations regarding Rho GTPases in fly development. First, multiple Rho GTPases appear to be involved in most of the individual events studied, and second, in most cases the phenotype observed is the disruption of normal actin structures, which leads to defects in cell-shape changes and migration. However, in light of more recent studies using loss-of-function (LOF) mutations in Rho1, Cdc42 and the three Rac genes, the first of these conclusions needs reconsideration. Not all of the phenotypes observed with expression of DN- or CA- versions of these proteins were corroborated by the lossof-function phenotypes. Furthermore, most of the phenotypes observed with the LOF mutations are specific to that Rho GTPase, suggesting that Rho, Cdc42, and the Rac genes may regulate distinct morphogenetic events during development, and that overexpression studies may sometimes overestimate the role of Rho GTPases in a given event. Nonetheless, the LOF studies bear out the second generalization: the loss-of-function phenotypes are likely the result of numerous defects in actin architecture in the mutants throughout development.

\section{Rho1}

Rhol was the first Rho GTPase for which LOF mutations were isolated in Drosophila (Magie et al. 1999, Strutt et al., 1997). Loss of Rho1 function is associated with both maternal and zygotic phenotypes. Reduction of Rho1 levels during oogenesis results in marked disruption of the actin cytoskeleton, particularly at the oocyte cortex and in the actin-rich ring canals. Whereas in general, the maternal role of zygotically lethal genes such as Rhol is studied by the construction of germline clones that lack functional Rho1, this approach proved impossible with Rhol due to its requirement for cell viability and/or proliferation. Perhaps reflecting the wellestablished requirement for Rho1 in cytokinesis, germline clones of Rho1 rapidly degenerate and no eggs are produced (CRM, unpublished observation). To circumvent this difficulty, studies of Rho1 in oogenesis have utilized genetic methods to reduce but not eliminate maternal Rho1 protein, mimicking a hypomorphic allele (Magie et al., 1999, 2002; Parkhurst and Ish-Horowicz, 1991). This technique is particularly useful for cell-essential genes such as Rho1, since it allows the study of processes requiring these gene products where this would otherwise be impossible with currently available technology.

Despite defects in actin architecture in egg chambers with reduced Rho1, these oocytes develop and produce embryos with several mutant phenotypes. Like embryos expressing DNRho1, reduced maternal Rho1 leads to defects in cellularization as evidenced by the presence of multinucleate cells at the blastoderm stage (Magie and Parkhurst, 2004). These embryos fail to maintain expression of the segment-polarity gene engrailed, resulting in segmentation defects. The maintenance of engrailed expression is known to be dependent on Wnt/Wingless and Hedgehog signaling between neighboring cells in the embryonic epidermis. While superficially this suggests a role for Rhol in the Wingless pathway, subsequent studies have shown that the underlying cause of this phenotype may result from a general defect in endocytosis and secretion in Rhol maternal mutants that leads to reduction of Wingless-containing vesicles (Magie and Parkhurst, 2004). Thus it is likely that this defect points either to the inability to properly 
internalize or export vesicles due to abnormal cortical actin structure, or to a direct role for Rho1 in the regulation of intracellular vesicle trafficking events. Both of these elements of vesicular trafficking events are regulated by Rho1 homologues in mammalian cells (cf. Qualmann and Mellor, 2003).

Embryos zygotically mutant for Rhol develop until late embryogenesis and exhibit several phenotypes distinct from both of the maternal phenotypes described above. Interestingly, the zygotic phenotypes of Rho1 LOF mutants are not identical to those of embryos expressing DNRho1. The major late-embryonic phenotype associated with expression of DN-Rhol is the failure of dorsal closure during embryogenesis, but zygotic Rhol LOF mutants do not have this phenotype (Genova et al., 2000; Magie et al., 1999). Rhol LOF mutants exhibit imperfect dorsal closure, characterized by misalignment of cells and disorganization of the actin cytoskeleton and actin cable at the leading edge, but these mutants invariably close the dorsal hole. The primary defect in Rhol zygotic LOF mutants, however, is failure of head involution due to defects in the cell migrations necessary to internalize the neural tissue, not to any defect in the required apoptotic events (Fig. 3B). In the case of Rho1, the discrepancy between the LOF and DN DC phenotypes cannot be explained by perdurance of maternal protein since Rho1 mutant embryos derived from germlines with reduced levels of Rhol do not exhibit a more severe DC phenotype (Magie et al., 1999). Thus the DN-Rho dorsal-open phenotype likely represents a cross-inhibition of Rac signaling pathways, for reasons discussed below.

Studies using LOF alleles to study the role of Rho1 in neurogenesis have indicated that Rho1 has a role in this process that is distinct from that suggested by expression of DN-Rhol. Generation of Rhol mutant clones in the CNS with the MARCM (Mosaic Analysis with a Repressible Cell Marker) technique, which utilizes the Gal80 co-repressor and a Gal4 driven reporter gene to mark mutant clones generated by Flp/FRT recombination, has indicated a role for Rho1 in neuroblast proliferation and dendritic complexity (Lee et al., 2000). Axon outgrowth, however, is unaffected, suggesting that the axon outgrowth defect in DN-Rho1 expressing neurons may be another example of Rac cross-inhibition (see below).

\section{Cdc42}

More recently, loss-of-function alleles of $\mathrm{Cdc} 42$ have been isolated, and studies utilizing these alleles have confirmed a requirement for $\mathrm{Cdc} 42$ throughout oogenesis and embryogenesis (Genova et al., 2000). Generation of follicle cell clones during oogenesis that are mutant for $C d c 42$ results in their loss of cuboidal shape and epithelial integrity (Genova et al., 2000). Loss of Cdc42 in the entire germline results in defects in actin cytoskeletal structure that are more subtle than those observed with the DN- or CA- versions. Moreover, while DN- and CA-Cdc42 expression in the germline results in complete failure of nurse cell dumping, Cdc42 mutant germlines exhibit only delayed nurse cell dumping.

The phenotype of zygotic Cdc42 LOF mutants differs somewhat from that associated with expression of DN-Cdc42 during embryogenesis, as Cdc42 LOF mutants do not exhibit failure of dorsal closure (Genova et al., 2000; Magie et al., 1999). Rather, these mutants fail to complete germ band retraction, and thus do not develop far enough to initiate dorsal closure at all. The possibility remains that $\mathrm{Cdc} 42$ does play a role in dorsal closure that is masked by the arrest of germ band retraction in these embryos. It is also interesting to note that expression of DN-Cdc42 does not result in a failure of germ band retraction, although perhaps induction of DN-Cdc42 at an earlier point in embryogenesis would have this effect. This discrepancy may reflect the varied effects of DN-Rho GTPases in different cell types and developmental contexts. Further 
investigation into $\mathrm{Cdc} 42$ function during embryogenesis, such as generation of $\mathrm{Cdc} 42$ mutant clones in the epithelium during dorsal closure to address the issue of whether germ band retraction failure masks a DC phenotype, will be of importance in deciphering its pleiotropic roles.

\section{Rac}

Because there are three Rac homologues in Drosophila, examination of the LOF phenotype of Rac required the generation of flies carrying mutations in Rac1, Rac2, and $m t l$ (triple Rac mutants), which was only accomplished recently (Hakeda-Suzuki et al., 2002). Initial studies of the Rac LOF phenotypes suggest that they mimic the phenotypes associated with expression of DN-Rac1 more closely than the Rhol and Cdc42 LOF phenotypes mirror DN- Rho and Cdc42 expression. Zygotic triple Rac LOF mutants, like embryos expressing DN-Rac1, fail to complete dorsal closure, resulting in a classical cuticle phenotype associated with DC defects: a large hole in the dorsal midzone (Fig. 3C). In these mutants, LE cells do not elongate properly and those cells do not exhibit the protrusive activity seen in wildtype (Hakeda-Suzuki et al., 2002). Elongation of the LE cells in early DC is associated with signaling through the JNK pathway, and thus Rac is the most likely candidate GTPase to act upstream of JNK in flies. To this point, however, no genetic or molecular evidence links Rac to other JNK pathway members in Drosophila, and whether the requirement of Rac for LE cell elongation reflects a role for Rac in JNK signaling or downstream of JNK pathway activation to effect cell-shape change is still largely an open question. However, the strong DC phenotype associated with removal of Rac activity suggests that the DC defects observed in DN-Rho and DN-Cdc42 embryos may represent at least a partial cross-inhibition of Rac signaling pathways.

The neurogenesis defects associated with expression of DN- or CA- Rac are also recapitulated in LOF mutant embryos. Rac triple mutants exhibit defects in both guidance and growth of axons (Ng et al., 2002), suggesting that Rac is the major Rho GTPase that regulates these processes. Interestingly, Rac triple mutants do not exhibit defects in planar cell polarity in the eye (HakedaSuzuki et al., 2002), and thus PCP phenotypes resulting from expression of DN-Rac1 may actually be attributable to impingement on Rhol or Cdc42 signaling.

\section{Downstream effectors of Rho GTPase signaling}

A major open question in the study of Rho GTPase function is how activation of these proteins can lead to such diverse effects in different cellular environments and developmental contexts. Although it has generally been assumed that each of the distinct cellular responses resulting from disruption of Rho signaling is due to loss of a specific downstream pathway, an alternate possibility is that the diversity of events requiring Rho GTPases reflects the ubiquitous role of the cytoskeleton. This possibility is supported by the potency of Rho and its homologues in regulating actin dynamics and by the fact that changes in cytoskeletal architecture have been shown to have far-reaching and pleiotropic effects.

Rho proteins act by binding to and activating downstream effector molecules, which then carry out the multitude of Rho functions. Presumably, the varied effects of Rho proteins result from activation of distinct downstream effectors in different contexts. Most of the currently known effectors were identified in cell culture using DN- and CA- Rho GTPases (see Table 1; reviewed in Bishop and Hall, 2000). Many of these effectors are kinases, most of which appear to more or less directly regulate actin dynamics. These include Protein Kinase N (PKN), which has been implicated in actin cytoskeletal regulation, cell adhesion and vesicle transport, and Rho- 
associated kinase (ROCK) (Amano et al., 1996; Kimura et al., 1996). ROCK regulates actinbased contractile events through phosphorylation of the nonmuscle myosin II regulatory light chain (MRLC), either directly or through activation of myosin light-chain kinase (MLCK). Another ROCK target is LIM kinase (LIMK), which acts to inhibit the actin severing protein cofilin following phosphorylation by ROCK (Maekawa et al., 1999). The Rho effector Citron kinase localizes to the cleavage furrow of dividing cells and, like ROCK, phosphorylates the MRLC, thus regulating the actin-myosin based contractility necessary for cytokinesis (Yamashiro et al., 2003). The lipid modifying kinase phosphatidylinositol-4-phosphate 5-kinase (PI4,5K) acts downstream of Rho and affects actin dynamics by producing PI4,5 $\mathrm{P}_{2}$, which can then uncap gelsolin to allow filament elongation or promote a conformational change in vinculin to allow it to tether actin at focal adhesions (Gilmore and Burridge, 1996; Janmey and Stossel, 1987). In addition, a number of Rho effectors are scaffold molecules whose functions are either unknown (i.e. Rhophilin) (Reid et al., 1996; Watanabe et al., 1996), or are de novo nucleators of actin filaments (i.e. mDia1, mDia2; Watanabe et al., 1999; Watanabe et al., 1997).

In addition to their profound effects on the actin cytoskeleton, cell culture studies utilizing expression of DN- and CA-Rho GTPases have linked these proteins to a variety of other processes, including cell-cycle progression, transcriptional activation, and cell-cell adhesion. Some of these apparent roles in transcriptional activation and cell cycle progression have since been shown to be indirect effects of changes in cellular actin architecture (see below). One notable exception to this is JNK-pathway activation, where Rac and Cdc42 appear to play prominent roles. Expression of CA-Cdc42 or Rac1 promotes signaling through the JNK pathway, which is thought to be responsible for their mitogenic effect in cultured cells (Olson et al., 1995). It should be noted that in Drosophila, the major developmental event mediated by the JNK pathway is dorsal closure. Thus, even in this case, the ultimate cellular response to Rac activation is cell migration, not $G_{1}$ progression, as is the case in cell culture. A number of studies have shown that Rho GTPases regulate cell-cell adhesion, but the mechanism of this regulation is still under investigation. Given the core requirement for actin polymerization in the maintenance of epithelial sheets (Vasioukhin et al., 2000), it would not be surprising if most of the adhesive events mediated by Rho GTPases were mainly regulated through localized changes in actin dynamics at junctions.

A better understanding of how Rho GTPases govern these various downstream functions is largely dependent on gaining a more complete picture of the effectors that act in these pathways and the mechanisms through which they are regulated. Another important question is how cellsurface receptors activate particular Rho proteins and lead to signaling through a specific pathway, underscoring the importance of assigning specificity to the large family of Rho GEFs. Although work done in cell culture has provided a great deal of information on various effectors and pathways working both upstream and downstream of Rho activation, these studies are vulnerable to lack of specificity due to their reliance on overexpression and the use of DN- and CA- constructs. One mechanism through which Rho activates its effectors upon binding to GTP involves the disruption of autoinhibitory intramolecular interactions (reviewed in Bishop and Hall, 2000). ROCK, PKN, and mDia all contain autoinhibitory domains whose activity is at least partly relieved upon Rho binding. In the case of mDia, autoinhibition is achieved by binding of the $\mathrm{C}$-terminal diaphanous autoinhibitory domain (DAD) to the N-terminal Rho binding domain (RBD). Binding of Rho to the RBD displaces the DAD, activating mDia presumably by allowing it to nucleate de novo actin polymerization. Consistent with this proposed mechanism, removal of the $\mathrm{N}$-terminal RBD results in a putative constitutively active 
protein (Palazzo et al., 2001). However, recent studies have cast doubt on this canonical model, as Rho fails to re-activate actin nucleation by the mDia $\mathrm{C}$-terminus after addition of an inhibiting $\mathrm{N}$-terminal portion of the protein. Future studies with full length formin proteins will be required to resolve this emerging controversy. For now, it is clear that DRF proteins are Rho effectors, although the mechanism of regulation by Rho remains elusive.

\section{Identification of Rho pathway components in vivo}

It is possible that some of the effectors identified through the use of these dominant alleles will not be specific to the GTPase of interest due to cross-inhibition or interference with other RhoGTPase-mediated signaling pathways. The isolation of LOF mutations in the fly Rho family members has allowed for a genetic approach to the dissection of Rho GTPase signaling pathways, and provides an organismal and developmental context in which to study these pathways. Many effectors identified in flies are similar or identical to those known from cell culture systems, although their in vivo roles sometimes differ from those defined in the cell culture studies. What is also notable is that many of these effectors exhibit tissue specificity and are necessary only for certain developmental events. One example that illustrates this point well is that of two formin-homology proteins, cappuccino (capu) and diaphanous (dia), that both interact with Rho in Drosophila. capu is zygotically dispensable, but homozygous capu mutant females are sterile. Females doubly heterozygous for mutations in Rhol and capu are also sterile and exhibit actin defects during oogenesis (Magie et al., 1999). dia is also required maternally, but for different developmental events during oogenesis and embryogenesis. dia mutants have follicle cell cytokinesis defects, and embryos lacking maternal dia fail to cellularize in a manner similar to DN-Rho1 injected embryos (Afshar et al., 2000; Crawford et al., 1998). Females transheterozygous for mutations in Rhol and dia exhibit maternal synthetic lethality (SMP, unpublished observation). Taken together, this suggests that Dia is required downstream of Rho1 at cellularization. Thus, different formin homology proteins appear to be required downstream of the same Rho GTPase at distinct developmental stages.

Additional studies have demonstrated roles for other effectors downstream of Rho GTPases in flies, all of which appear to be required only for particular developmental events. The observation that Rhol mutants exhibit mis-localization of the adherens junction component ECadherin during DC lead to the identification of two novel binding partners of Drosophila Rho1, p120 catenin and $\alpha$-catenin, both components of adherens junctions (Magie et al., 2002). In the case of p120 catenin, this link is consistent with studies in mammalian systems (Anastasiadas and Reynolds, 2000). The fly homologue of PKN has been shown to act downstream of Rho1 in mediating cell-shape change at the leading edge during DC (Lu and Settleman, 1999). Studies utilizing a LOF allele of Drok, the Drosophila homologue of mammalian ROCK, have demonstrated a requirement for this Rho1 effector in planar cell polarity downstream of the Frizzled receptor (Winter et al., 2001). Drok also mediates the effects of Rho1 on dendritic morphogenesis in developing neurons (Lee et al., 2000). A LIM kinase homologue was recently identified in flies and shown to be required for regulating morphogenetic events in response to ecdysone signaling (Chen et al., 2004). Mutations in blistered, the fly homologue of mammalian SRF, can suppress defects in wing morphogenesis resulting from overexpression of Dlimk, demonstrating that SRF mediated transcriptional activation downstream of Rho is conserved in Drosophila (Chen et al., 2004).

Drosophila genetics has also proved useful in identifying upstream components of Rho signaling pathways. A major hurdle encountered in searching for GEFs that activate Rho 
GTPases in response to particular stimuli is lack of specificity: many of these proteins appear capable of activating several Rho family members. The power of a genetic approach stems from the ability to identify specific GEFs by screening for genetic interactions with a given Rho family member. Analysis of the resulting phenotype can often give some indication of when and where a particular GEF is required. This approach has been particularly successful in identifying the Rho-specific GEF pebble (discussed above; Hime and Saint, 1992; Prokopenko et al., 1999). Another Rho1 GEF, DRhoGEF2, was identified in flies based in homology to mammalian Rhospecific GEFs. DRhoGEF2 mutants, like embryos expressing DN-Rho1, fail to form a ventral furrow, and exhibit little of the apical flattening of cells in the ventral region that is observed in wildtype embryos (Barrett et al., 1997; Hacker and Perrimon, 1998). A third GEF, Trio, was recently shown to act upstream of Rac signaling in neuronal morphogenesis, but is not required during DC (Hakeda-Suzuki et al., 2002).

Two additional upstream activators of Rho1 have been identified in Drosophila, and both are involved in G-protein coupled signaling. Concertina encodes the $\alpha$ subunit of a heterotrimeric G-protein complex, and interacts dominantly with Rhol to produce egg chambers with abnormal F-actin structure and distribution, as well as poor cell-cell adhesion. However, unlike germlines doubly heterozygous for Rhol and capu, this interaction does not result in embryonic lethality (Magie et al., 1999). The receptor and ligand responsible for activating Concertina during oogenesis are currently unknown. More recently, the G-protein coupled receptor Tre1 was shown to be required for the trans-epithelial migration of germ cells that occurs during gastrulation (Kunwar et al., 2003). Expression of DN-Rho1 results in a similar phenotype to loss of Tre1 function, suggesting that Tre1 may act upstream of Rho1 in directing this migration. The success of these studies points to the power of genetics in identifying tissue and pathway-specific upstream activators of Rho GTPase signaling.

Although initial genetic studies in flies have been highly successful in building pathways through which Rho GTPases regulate various developmental events, our understanding of how these proteins carry out their many functions is still incomplete. Our lab has recently conducted a screen of chromosomal deficiencies encompassing $85 \%$ of the Drosophila genome in order to identify regions exhibiting dominant genetic interactions with Rhol LOF alleles. Of the 250 deficiencies tested, 22 exhibited dominant interactions with Rhol, and are thus likely to contain genes that encode members of various pathways involving Rho1. By testing these deficiencies with known Rhol interactors, we can begin to build up these pathways from genetic data. Using the complete Drosophila genome, it is possible to identify candidate genes within these regions that are likely Rho1 interactors based on known function or homology to other proteins. Now that LOF mutations in the three fly Racs and Cdc42 are also available, similar screens could be carried out to identify effectors and upstream activators for the other Rho family members.

\section{Conclusion and future directions}

Rho GTPases have been linked to a large number of cellular processes. A question central to an accurate understanding of Rho biology is how directly it affects each of these processes. Evidence is accumulating that Rho proteins may in fact have one or two major functions, with an ability to affect others secondarily (Fig. 4). The ability of Rho to influence transcriptional activation through SRF, for example, is due not to a direct effect on transcription, but rather to alteration of the levels of cellular G-actin that occurs as a result of activation of mDia1 and ROCK downstream of Rho (Sotiropoulos et al., 1999). Specifically, SRF responds to lower Gactin levels by activating transcription from its target loci. Studies investigating the links 
between Rho and cell cycle control have also implicated actin cytoskeletal regulation as the underlying mechanism. Disruption of stress fibers in fibroblasts results in a $\mathrm{G}_{1}$ arrest due to the failure to sustain ERK signaling and subsequent CyclinD1 induction (Roovers and Assoian, 2003). Indeed, a recent study links RhoA, acting through mDia and ROCK, to cell cycle control through the detection of cell shape changes (Mammoto, 2003). Thus, some of the most wellstudied instances of Rho GTPases activating transcription are turning out to be secondary to defects in the actin cytoskeleton. It is possible that, as other transcriptional events downstream of Rho, Rac, and Cdc42 continue to be studied in greater mechanistic detail, these will also be similarly indirect.

To date, most studies done in Drosophila appear to support this more "actin-centric" view of Rho signaling (Fig. 4B). The processes regulated by Rho effectors in Drosophila can in many cases be traced back to their direct effects on actin dynamics or other closely related processes (Table 1). dRok regulates PCP through the myosins zipper and crinkled (Winter et al., 2001). PKN is involved in DC and has been implicated in cell adhesion and vesicle transport in other systems ( $\mathrm{Lu}$ and Settleman, 1999; Mukai, 2003). Dia and Capu, as members of the formin homology class of proteins, likely nucleate actin filament assembly like their mammalian and yeast homologues. The Rho binding protein $\alpha$-catenin is a key element linking the actin cytoskeleton to adherens junctions (Knust and Bossinger, 2002; Tepass et al., 2001).

A precise determination of the pathways activated by Rho GTPases and the relationships of those pathways to others in the cell is crucial to determining the biological function of these important signaling proteins. The ease of manipulation and flexibility of the cell culture system has provided a wealth of information on the function of Rho GTPases, and has allowed for the identification of many upstream activators and downstream effectors of Rho signaling. However, cell culture studies cannot fully address the function of these proteins in a developmental or organismal context. Drosophila development provides an ideal setting in which to add to existing biochemical and cell culture studies by addressing these questions. The smaller size of the Rho family and relative simplicity and speed of doing genetics in flies simplify the complex task of identifying pathway members. We expect the ongoing candidate gene studies based on available data from mammalian cell culture will add to our understanding of how Rho functions in metazoan development. Genetic screens will allow for the identification of novel Rho pathway members, and immediately provide an organismal system in which to study them. Drosophila promises to be an excellent complement to mammalian cell culture in developing a more global understanding of Rho biology.

\section{Acknowledgements}

We thank Darren Kamikura, Jeremy Nance, Amir Oryan and members of the lab for discussion and comments on the manuscript. Work in our lab is supported by NIH grant GM066847 (to S.M.P). 


\section{References}

Adler, P.N. 2002. Planar signaling and morphogenesis in Drosophila. Dev. Cell. 2: 525-535.

Afshar, K., B. Stuart, and S. Wasserman. 2000. Functional analysis of the Drosophila

Diaphanous FH protein in early embryonic development. Development 127: 1887-1897.

Bagrodia, S., B. Derijard, R.J. Davis, and R.A. Cerione. 1995. Cdc42 and PAK-mediated signaling leads to Jun kinase and p38 mitogen-activated protein kinase activation. J. Biol. Chem. 270: 27995-27998.

Barrett, K., M. Leptin, and J. Settleman. 1997. The Rho GTPase and a putative RhoGEF mediate a signaling pathway for the cell shape changes in Drosophila gastrulation. Cell 91: 905-915.

Bishop, A.L., and A. Hall. 2000. Rho GTPases and their effector proteins. Biochem. J. 348: 241255.

Boquet, P. 1999. Bacterial toxins inhibiting or activating small GTP-binding proteins. Ann. N.Y. Acad. Sci. 886: 83-90.

Braga, V.M.M., A. DelMaschio, L. Machesky, E. Dejann. 1999. Regulation of cadherin function by Rho and Rac: modulation by junction maturation and cellular context. Mol. Biol. Cell. 10: 9-22.

Burridge, K., and K. Wennerberg. 2004. Rho and Rac take center stage. Cell 116: 167-179.

Coleman, M.L., and M.F. Olson. 2002. Rho GTPase signalling pathways in the morphological changes associated with apoptosis. Cell Death Differ. 9: 493-504.

Coso, O.A., M. Chiariello, J.C. Yu, H. Teramoto, P. Crespo, N. Xu, T. Miki, and J.S. Gutkind. 1995. The small GTP-binding proteins Rac1 and Cdc42 regulate the activity of the JNK/SAPK signaling pathway. Cell 81: 1137-1146.

Crawford, J.M., N. Harden, T. Leung, L. Lim, and D.P. Kiehart. 1998. Cellularization in Drosophila melanogaster is disrupted by the inhibition of rho activity and the activation of Cdc42 function. Dev. Biol. 204: 151-164.

Eaton, S., R. Wepf, and K. Simons. 1996. Roles for Rac1 and Cdc42 in planar polarization and hair outgrowth in the wing of Drosophila. J. Cell. Biol. 135: 1277-1289.

Ellis, S., and H. Mellor. 2000. Regulation of endocytic traffic by rho family GTPases. Trends Cell Biol. 10: 85-88.

Etienne-Manneville, S., and A. Hall. 2002. Rho GTPases in cell biology. Nature 420: 629-635.

Fanto, M., U. Weber, D.I. Strutt, and M. Mlodzik. 2000. Nuclear signaling by Rac and Rho GTPases is required in the establishment of epithelial planar polarity in the Drosophila eye. Curr. Biol. 10: 979-988.

Foe, V.E. 1989. Mitotic domains reveal early commitment of cells in Drosophila embryos. Development 107: 1-22.

Fukata, M., and K. Kaibuchi. 2001. Rho-family GTPases in cadherin-mediated cell-cell adhesion. Nat. Rev. Mol. Cell Biol. 2: 887-897.

Genova, J.L., S. Jong, J.T. Camp, and R.G. Fehon. 2000. Functional analysis of Cdc42 in actin filament assembly, epithelial morphogenesis, and cell signaling during Drosophila development. Dev Biol. 221: 181-194.

Gilmore, A.P., and K. Burridge. 1996. Regulation of vinculin binding to talin and actin by phosphatidyl-inositol-4-5-bisphosphate. Nature 381: 531-535.

Glise, B., H. Bourbon, and S. Noselli. 1995. hemipterous encodes a novel Drosophila MAP kinase kinase, required for epithelial cell sheet movement. Cell 83: 451-461.

Glise, B., and S. Noselli. 1997. Coupling of Jun amino-terminal kinase and Decapentaplegic signaling pathways in Drosophila morphogenesis. Genes Dev. 11: 1738-1747. 
Hacker, U., and N. Perrimon. 1998. DRhoGEF2 encodes a member of the Dbl family of oncogenes and controls cell shape changes during gastrulation in Drosophila. Genes Dev. 12: 274-284.

Hakeda-Suzuki, S., J. Ng, J. Tzu, G. Dietzl, Y. Sun, M. Harms, T. Nardine, L. Luo, and B.J. Dickson. 2002. Rac function and regulation during Drosophila development. Nature 416: 438-442.

Hall, A. 1998. Rho GTPases and the Actin Cytoskeleton. Science 279: 509-514.

Harden, N., J. Lee, H.Y. Loh, Y.M. Ong, I. Tan, T. Leung, E. Manser, and L. Lim. 1996. A Drosophila homolog of the Rac- and Cdc42-activated serine/threonine kinase PAK is a potential focal adhesion and focal complex protein that colocalizes with dynamic actin structures. Mol. Cell. Biol. 16: 1896-1908.

Harden, N., H.Y. Loh, W. Chia, and L. Lim. 1995. A dominant inhibitory version of the small GTP-binding protein Rac disrupts cytoskeletal structures and inhibits developmental cell shape changes in Drosophila. Development 121: 903-914.

Harden, N., M. Ricos, Y.M. Ong, W. Chia, and L. Lim. 1999. Participation of small GTPases in dorsal closure of the Drosophila embryo: distinct roles for Rho subfamily proteins in epithelial morphogenesis. J. Cell Sci. 112: 273-284.

Hariharan, I.K., K.Q. Hu, H. Asha, A. Quintanilla, R.M. Ezzell, and J. Settleman. 1995. Characterization of rho GTPase family homologues in Drosophila melanogaster: overexpressing Rhol in retinal cells causes a late developmental defect. EMBO J. 14: 292302.

Hornstein, I., A. Alcover, and S. Katzav. 2004. Vav proteins, masters of the world of cytoskeleton organization. Cell Signal. 16: 1-11.

Jacinto, A., W. Wood, T. Balayo, M. Turmaine, A. Martinez-Arias, and P. Martin. 2000. Dynamic actin-based epithelial adhesion and cell matching during Drosophila dorsal closure. Curr. Biol. 10: 1420-1426.

Jacinto, A., W. Wood, S. Woolner, C. Hiley, L. Turner, C. Wilson, A. Martinez-Arias, and P. Martin. 2002. Dynamic analysis of actin cable function during Drosophila dorsal closure. Curr Biol. 12: 1245-1250.

Janmey, P.A., and T.P. Stossel. 1987. Modulation of gelsolin function by phosphatidylinositol 4,5-bisphosphate. Nature 325: 362-364.

Kjoller, L., and A. Hall. 1999. Signaling to Rho GTPases. In Exp Cell Res. Vol. 253. 166-79.

Kozma, R., S. Ahmed, A. Best, and L. Lim. 1995. The Ras-related protein Cdc42Hs and bradykinin promote formation of peripheral actin microspikes and filopodia in Swiss 3T3 fibroblasts. Mol. Cell. Biol. 15: 1942-1952.

Kozma, R., S. Sarner, S. Ahmed, and L. Lim. 1997. Rho family GTPases and neuronal growth cone remodelling: relationship between increased complexity induced by Cdc42Hs, Rac1, and acetylcholine and collapse induced by RhoA and lysophosphatidic acid. Mol. Cell. Biol. 17: 1201-1211.

Lee, T., C. Winter, S.S. Marticke, A. Lee, and L. Luo. 2000. Essential roles of Drosophila RhoA in the regulation of neuroblast proliferation and dendritic but not axonal morphogenesis. Neuron 25: 307-316.

Lu, Y., and J. Settleman. 1999. The Drosophila Pkn protein kinase is a Rho/Rac effector target required for dorsal closure during embryogenesis. Genes Dev. 13: 1168-1180. 
Luo, L., Y.J. Liao, L.Y. Jan, and Y.N. Jan. 1994. Distinct morphogenetic functions of similar small GTPases: Drosophila Drac1 is involved in axonal outgrowth and myoblast fusion. Genes Dev. 8: 1787-1802.

Mackay, D.J., and A. Hall. 1998. Rho GTPases. J. Biol. Chem. 273: 20685-20688.

Maekawa, M., T. Ishizaki, S. Boku, N. Watanabe, A. Fujita, A. Iwamatsu, T. Obinata, K. Ohashi, K. Mizuno, and S. Narumiya. 1999. Signaling from Rho to the actin cytoskeleton through protein kinases ROCK and LIM-kinase. Science 285: 895-898.

Magie, C.R., M.R. Meyer, M.S. Gorsuch, and S.M. Parkhurst. 1999. Mutations in the Rho1 small GTPase disrupt morphogenesis and segmentation during early Drosophila development. Development 126: 5353-5364.

Maruta, H. 1998. GTPase Regulators: GAPs, GDSs, and GDIs. In G Proteins, Cytoskeleton and Cancer. K. Kohama, editor. R.G. Landes Company, Austin, TX. pp.151-170.

Mazumdar, A., and M. Mazumdar. 2002. How one becomes many: blastoderm cellularization in Drosophila melanogaster. Bioessays 24: 1012-1022.

Minden, A., A. Lin, F.X. Claret, A. Abo, and M. Karin. 1995. Selective activation of the JNK signaling cascade and c-Jun transcriptional activity by the small GTPases Rac and Cdc42Hs. Cell 81: 1147-1157.

Mlodzik, M. 2002. Planar cell polarization: do the same mechanisms regulate Drosophila tissue polarity and vertebrate gastrulation? Trends Genet. 18: 564-571.

Mukai, H. 2003. The structure and function of PKN, a protein kinase having a catalytic domain homologous to that of PKC. J. Biochem. 133: 17-27.

Murphy, A.M., and D.J. Montell. 1996. Cell type-specific roles for Cdc42, Rac, and RhoL in Drosophila oogenesis. J. Cell Biol. 133: 617-630.

Ng, J., T. Nardine, M. Harms, J. Tzu, A. Goldstein, Y. Sun, G. Dietzl, B.J. Dickson, and L. Luo. 2002. Rac GTPases control axon growth, guidance and branching. Nature 416: 442-447.

Nobes, C.D., and A. Hall. 1995. Rho, rac, and cdc42 GTPases regulate the assembly of multimolecular focal complexes associated with actin stress fibers, lamellipodia, and filopodia. Cell 81: 53-62.

Noselli, S. 1998. JNK signaling and morphogenesis in Drosophila. Trends Genet. 14: 33-38.

Palazzo, A.F., T.A. Cook, A.S. Alberts, and G.G. Gundersen. 2001. mDia mediates Rhoregulated formation and orientation of stable microtubules. Nat. Cell Biol. 3: 723-729.

Prokopenko, S.N., A. Brumby, L. O'Keefe, L. Prior, Y. He, R. Saint, and H.J. Bellen. 1999. A putative exchange factor for Rho1 GTPase is required for initiation of cytokinesis in Drosophila. Genes Dev. 13: 2301-2314.

Qualmann, B., and H. Mellor. 2003. Regulation of endocytic traffic by Rho GTPases. Biochem. J. 371: 233-241.

Reid, T., T. Furuyashiki, T. Ishizaki, G. Watanabe, N. Watanabe, K. Fujisawa, N. Morii, P. Madaule, and S. Narumiya. 1996. Rhotekin, a new putative target for Rho bearing homology to a serine/threonine kinase, PKN, and rhophilin in the rho-binding domain. J. Biol. Chem. 271: $13556-13560$.

Ridley, A.J., and A. Hall. 1992. The small GTP-binding protein rho regulates the assembly of focal adhesions and actin stress fibers in response to growth factors. Cell 70: 389-399.

Ridley, A.J., H.F. Paterson, C.L. Johnston, D. Diekmann, and A. Hall. 1992. The small GTPbinding protein rac regulates growth factor-induced membrane ruffling. Cell 70: 401-410. 
Riesgo-Escovar, J.R., M. Jenni, A. Fritz, and E. Hafen. 1996. The Drosophila Jun-N-terminal kinase is required for cell morphogenesis but not for DJun-dependent cell fate specification in the eye. Genes Dev. 10: 2759-2768.

Roovers, K., and R.K. Assoian. 2003. Effects of rho kinase and actin stress fibers on sustained extracellular signal-regulated kinase activity and activation of $G(1)$ phase cyclin-dependent kinases. Mol. Cell. Biol. 23: 4283-4294.

Sahai, E., and C.J. Marshall. 2002. RHO-GTPases and cancer. Nat. Rev. Cancer 2: 133-142.

Settleman, J. 1999. Rho GTPases in development. Prog. Mol. Subcell. Biol. 22: 201-229.

Sotiropoulos, A., D. Gineitis, J. Copeland, and R. Treisman. 1999. Signal-regulated activation of serum response factor is mediated by changes in actin dynamics. Cell 98: 159-169.

Strutt, D.I., U. Weber, and M. Mlodzik. 1997. The role of RhoA in tissue polarity and Frizzled signalling. Nature 387: 292-295.

Valster, A.H., P.K. Hepler, and J. Chernoff. 2000. Plant GTPases: the Rhos in bloom. Trends Cell Biol. 10: 141-146.

Vasioukhin, V., C. Bauer, M. Yin, and E. Fuchs. 2000. Directed actin polymerization is the driving force for epithelial cell-cell adhesion. Cell 100: 209-219.

Watanabe, G., Y. Saito, P. Madaule, T. Ishizaki, K. Fujisawa, N. Morii, H. Mukai, Y. Ono, A. Kakizuka, and S. Narumiya. 1996. Protein kinase N (PKN) and PKN-related protein rhophilin as targets of small GTPase Rho. Science 271: 645-648.

Watanabe, N., T. Kato, A. Fujita, T. Ishizaki, and S. Narumiya. 1999. Cooperation between mDial and ROCK in Rho-induced actin reorganization. Nat. Cell. Biol. 1: 136-143.

Watanabe, N., P. Madaule, T. Reid, T. Ishizaki, G. Watanabe, A. Kakizuka, Y. Saito, K. Nakao, B.M. Jockusch, and S. Narumiya. 1997. p140mDia, a mammalian homolog of Drosophila diaphanous, is a target protein for Rho small GTPase and is a ligand for profilin. EMBO J. 16: 3044-3056.

Yamashiro, S., G. Totsukawa, Y. Yamakita, Y. Sasaki, P. Madaule, T. Ishizaki, S. Narumiya, and F. Matsumura. 2003. Citron kinase, a Rho-dependent kinase, induces di-phosphorylation of regulatory light chain of myosin II. Mol. Biol. Cell 14: 1745-1756. 


\section{Figure Legends}

Figure 1. The Rho GTPase cycle. Rho GTPases function as molecular switches, with active, GTP-bound and inactive, GDP-bound forms. Associated regulatory enzymes modulate their position in this cycle: Guanine-nucleotide Exchange Factors (GEFs) activate Rho by catalyzing the exchange of GDP for GTP, GTPase-Activating Proteins (GAPs) inactivate Rho by increasing the rate at which it hydrolyzes GTP, and Guanine-Dissociation Inhibitors (GDIs) keep Rho in a GDP-bound, inactive state. The activation of G-protein coupled receptors is thought to lead to the activation of particular GEFs and the subsequent activation of Rho. Once Rho is active, it can bind effector molecules and carry out downstream functions.

Figure 2. Drosophila development. (A-B'") Oogenesis. (A) Schematic of a stage 10 egg chamber, consisting of the germline nurse cells (yellow) and oocyte (red), surrounded by somatic follicle cells (blue). The nurse cells and oocyte are connected by actin-rich ring canals that allow for the transport of materials from cell to cell. The border cells are a specialized subset of follicle cells that migrate to the anterior of the oocyte and are responsible for forming the micropyle, the sperm entry point. Anterior is left. (B) Wildtype stage 10 egg chamber stained with phalloidin to label F-actin. Frontal (B') and edge (B") views of a ring canal. (B"') Actin structure of the oocyte cortex and follicle cells. Images after Magie et al., 1999.

(C-D) Cleavage and cellularization in Drosophila. (C) The stages of nuclear migration, proceeding from early (top) to late (bottom). The nuclear cycles comprising each stage are indicated to the right. Cleavage divisions occur within a syncytium. Nuclei begin in the anterior cytoplasm and migrate to the poles as they divide. Once nuclei reach the surface (cycle 10), the pole cells (germline anlage) form (middle image). Cellularization occurs during the 14th cell cycle following fertilization. Anterior is left. (D) Cellularization involves the invagination of the plasma membrane between nuclei (blue) and the coordinated regulation of actin (red) and myosin (green).

(E) SEMs of the successive stages of Drosophila gastrulation. (i) Cellular blastoderm. (ii) Gastrulation begins with ventral furrow formation (not shown) and germband extension. Arrowheads indicate transverse folds, arrow indicates the cephalic furrow. (iii-iv) Germband extension continues until the germband has fully migrated along the dorsal surface of the embryo. ( $v$-vi) Germband retraction results in a dorsal hole in the embryonic epithelium. (vii) Dorsal closure zips the lateral epithelia together at the dorsal midline, while head involution internalizes anterior head structures. (viii) A fully-formed larva ready to hatch.

Figure 3. Rho GTPases in Drosophila. These images show the surface of the larval cuticle, which is indicative of underlying patterning established during embryogenesis. Cuticle phenotype of wildtype (A), Rhol loss-of-function (B), and dominant negative Rho1 (C) photographed with darkfield (A-B) or phase (C) microscopy. The dorsal anterior hole in the Rhol LOF larval cuticle (arrowheads in B) is a result of the inability of brain tissue, which is not internalized properly, to secrete cuticle. Overexpression of constitutively-active (CA) or dominant-negative (DN) forms of Rho GTPase proteins results in a distinct dorsal open phenotype resulting from failed dorsal closure, with the dorsal hole located more posteriorly than 
in B (arrows in C). Images after Magie et al., 1999. Anterior is left; the ventral surface is shown.

Figure 4. Possible mechanisms of Rho function. (A) Rho acts directly through a number of distinct signaling pathways to carry out its downstream functions. Actin cytoskeletal regulation is one function among many. This is the classical view of Rho function. (B) Rho acts primarily as a regulator of the actin cytoskeleton, and its links to other cellular functions are indirect. 
Table 1. Downstream effectors of Rho GTPases.

\begin{tabular}{|c|c|c|c|c|c|c|}
\hline \multirow[t]{2}{*}{ effector } & \multirow[t]{2}{*}{ protein class } & \multirow[t]{2}{*}{ functions } & \multicolumn{3}{|c|}{ spec ificity } & \multirow{2}{*}{ references } \\
\hline & & & Rho & Rac & Cdc42 & \\
\hline Rho-kinase (ROCK) & ser/thr kinase & actin/myosin interactions & $\sqrt{ }$ & & & Kimura et al. (1996) \\
\hline $\begin{array}{l}\text { Protein kinase N (PKN) } \\
\text { /PRK1, PRK2 }\end{array}$ & ser/thr kinase & $\begin{array}{l}\text { actin regulation } \\
\text { actin/myosin interactions } \\
\text { cell adhesion } \\
\text { vesicle transport }\end{array}$ & $\sqrt{ }$ & & & Amano et al. (1996) \\
\hline Citron kinase & ser/thr kinase & cytokinesis & $\sqrt{ }$ & & & Madule et al. (1995) \\
\hline PAK1, 2, 3, 4 & ser/thr kinase & $\begin{array}{l}\text { JNK signaling } \\
\text { actin regulation }\end{array}$ & & $\sqrt{ }$ & $\sqrt{ }$ & Bagrodia and Cerione (1999) \\
\hline Mlk2, 3 & ser/thr kinase & JNK signaling & & $\sqrt{ }$ & $\sqrt{ }$ & Teramoto et al. (1996) \\
\hline MEKK1, 4 & ser/thr kinase & JNK signaling & & $\sqrt{ }$ & $\sqrt{ }$ & Fanger et al. (1997) \\
\hline p70 S6 kinase & ser/thr kinase & translation regulation & & $\sqrt{ }$ & $\sqrt{ }$ & Chou and Blenis (1996) \\
\hline $\operatorname{MRCK} \alpha, \beta$ & ser/thr kinase & actin regulation & & & $\sqrt{ }$ & Leung et al. (1998) \\
\hline Ack1, 2 & tyr kinase & unknown & & & $\sqrt{ }$ & Manser et al. (1993) \\
\hline MBS & phosphatase & actin/myosin interactions & $\sqrt{ }$ & & & Kimura et al. (1996) \\
\hline $\begin{array}{l}\text { Phosphatidylinositol-4- } \\
\text { phosphate 5-kinase } \\
\text { (PI-4-P5K) }\end{array}$ & lipid kinase & $\begin{array}{l}\mathrm{PIP}_{2} \text { levels } \\
\text { actin regulation }\end{array}$ & $\sqrt{ }$ & $\sqrt{ }$ & & Ren et al. (1996) \\
\hline $\begin{array}{l}\text { Phosphatidylinositol-3 } \\
\text { kinase (PI-3-K) }\end{array}$ & lipid kinase & $\mathrm{PIP}_{3}$ levels & & $\sqrt{ }$ & $\sqrt{ }$ & Tolias et al. (1995) \\
\hline Phospholipase-D (PLD) & lipid kinase & PA levels & $\sqrt{ }$ & $\sqrt{ }$ & $\sqrt{ }$ & Malcolm et al. (1996) \\
\hline Synaptojanin 2 & lipid phosphatase & endocytosis & & $\sqrt{ }$ & & Malecz et al. (2000) \\
\hline $\begin{array}{l}\text { Diacylglycerol (DAG) } \\
\text { kinase }\end{array}$ & lipase & $\begin{array}{l}\text { PA levels } \\
\text { cytokinesis }\end{array}$ & $\sqrt{ }$ & $\sqrt{ }$ & & Tolias et al. (1998) \\
\hline $\begin{array}{l}\text { Phospholipase-C- } \beta 2 \\
\text { (PLC- } \beta 2)\end{array}$ & lipase & $\mathrm{DAG} / \mathrm{IP}_{3}$ levels & & $\sqrt{ }$ & $\sqrt{ }$ & Illenberger et al. (1998) \\
\hline Rhophilin & scaffold & actin regulation & $\sqrt{ }$ & & & Peck et al. (2002) \\
\hline Rhotekin & scaffold & unknown & $\sqrt{ }$ & & & Reid et al. (1996) \\
\hline mDia1, mDia2 & scaffold & actin/microtubule regulation & $\sqrt{ }$ & & & Watanabe et al. (1997) \\
\hline Kinectin & scaffold & kinesin binding & $\sqrt{ }$ & $\sqrt{ }$ & & Hotta et al. (1996) \\
\hline p67PHOX & scaffold & NADPH oxidase & & $\sqrt{ }$ & & Abo et al. (1991) \\
\hline POR-1 & scaffold & actin regulation & & $\sqrt{ }$ & & Van Aelst et al. (1996) \\
\hline p140Sra-1 & scaffold & actin regulation & & $\sqrt{ }$ & & Kobayashi et al. (1998) \\
\hline WAVE/ Scar & scaffold & actin regulation & & $\sqrt{ }$ & & Miki et al. (1998) \\
\hline $\mathrm{POSH}$ & scaffold & unknown & & $\sqrt{ }$ & & Tapon et al. (1998) \\
\hline IQGAP1, 2 & scaffold & actin/cell-cell contacts & & $\sqrt{ }$ & $\sqrt{ }$ & Kaibuchi et al. (1999) \\
\hline Par6 & scaffold & cell-cell contacts & & & $\sqrt{ }$ & Joberty et al. (2000) \\
\hline WASP/N-WASP & scaffold & actin regulation & & & $\sqrt{ }$ & Rohatgi et al. (1999) \\
\hline CIP-4 & scaffold & unknown & & & $\sqrt{ }$ & Aspenstrom (1997) \\
\hline Borg1-5 & scaffold & actin regulation & & & $\sqrt{ }$ & Joberty et al. (1999) \\
\hline SPEC1, 2 & scaffold & $\begin{array}{l}\text { JNK signaling } \\
\text { actin regulation }\end{array}$ & & $\sqrt{ }$ & $\sqrt{ }$ & Pirone et al. (2000) \\
\hline Plexin-B1 & semaphorin receptor & actin regulation & & $\sqrt{ }$ & & Vikis et al. (2000) \\
\hline$n$-Chimaerin & GAP & actin regulation & & $\sqrt{ }$ & $\sqrt{ }$ & Kozma et al. (1996) \\
\hline
\end{tabular}


Table 2. Processes linked to Rho GTPase activity in Drosophila.

\begin{tabular}{|c|c|c|c|c|}
\hline & constitutively-active & dominant-negative & loss-of-function & references \\
\hline Rho & dorsal closure (mild) & $\begin{array}{l}\text { dorsal closure (failure) } \\
\quad \text { (JNK pathway compromised) } \\
\text { leading edge cell shape changes } \\
\text { ventral furrow formation } \\
\text { cytokinesis }\end{array}$ & $\begin{array}{l}\text { dorsal closure (mild) } \\
\quad \text { (no JNK pathway effects) } \\
\text { dendrite morphology } \\
\text { planar cell polarity } \\
\text { cytokinesis } \\
\text { head involution } \\
\text { actin structure in oogenesis } \\
\text { segmentation }\end{array}$ & $\begin{array}{l}\text { Barrett et al. (1997) } \\
\text { Strutt et al. (1997) } \\
\text { Crawford et al. (1998) } \\
\text { Hacker and Perrimon (1998) } \\
\text { Harden et al. (1999) } \\
\text { Lu and Settleman (1999) } \\
\text { Magie et al. (1999) } \\
\text { Lee et al. (2000) } \\
\text { Bloor and Kiehart (2002) }\end{array}$ \\
\hline Rac & $\begin{array}{l}\text { axon outgrowth } \\
\text { PCP } \\
\text { dorsal closure } \\
\quad \text { (amnioserosa contraction) } \\
\text { ectopic JNK activation }\end{array}$ & $\begin{array}{l}\text { axon outgrowth } \\
\text { wing hair placement } \\
\text { planar cell polarity } \\
\text { dorsal closure (failure) } \\
\text { germband retraction } \\
\text { head involution } \\
\text { ectopic cell death } \\
\text { border cell migration }\end{array}$ & $\begin{array}{l}\text { axon outgrowth } \\
\text { dorsal closure (failure) } \\
\text { myoblast fusion }\end{array}$ & $\begin{array}{l}\text { Luo et al. (1994) } \\
\text { Eaton et al. (1995) } \\
\text { Harden et al. (1995) } \\
\text { Eaton et al. (1996) } \\
\text { Murphy and Montell (1996) } \\
\text { Glise and Noselli (1997) } \\
\text { Harden et al. (1999) } \\
\text { Hakeda-Suzuki et al. (2002) } \\
\text { Harden et al. (2002) } \\
\text { Ng et al. (2002) }\end{array}$ \\
\hline Cdc42 & $\begin{array}{l}\text { dorsal closure (mild) } \\
\text { nurse cell fusion } \\
\text { cytokinesis } \\
\text { ectopic JNK activation }\end{array}$ & $\begin{array}{l}\text { dorsal closure (failure) } \\
\text { nurse cell fusion } \\
\text { wing hair morphology } \\
\text { wing disc cell shape }\end{array}$ & $\begin{array}{l}\text { germband retraction } \\
\text { epithelial integrity } \\
\text { follicle cell epithelial morphology } \\
\text { actin morphology in oogenesis } \\
\text { no JNK pathway effects }\end{array}$ & $\begin{array}{l}\text { Eaton et al. (1995) } \\
\text { Eaton et al. (1996) } \\
\text { Murphy and Montell (1996) } \\
\text { Riesgo-Escovar et al. (1996) } \\
\text { Glise and Noselli (1997) } \\
\text { Crawford et al. (1998) } \\
\text { Harden et al. (1999) } \\
\text { Ricos et al. (1999) } \\
\text { Genova et al. (2000) }\end{array}$ \\
\hline RhoL & $\begin{array}{l}\text { nurse cell-follicle cell contacts } \\
\text { follicle cell epithelial morphology }\end{array}$ & $\begin{array}{l}\text { nurse cell-follicle cell contacts } \\
\text { nurse cell fusion }\end{array}$ & mutant not yet reported & Murphy and Montell (1996) \\
\hline RhoBTB & no data & no data & no data & N/A \\
\hline
\end{tabular}


Figure 1.

(Johndrow, Magie \& Parkhurst)

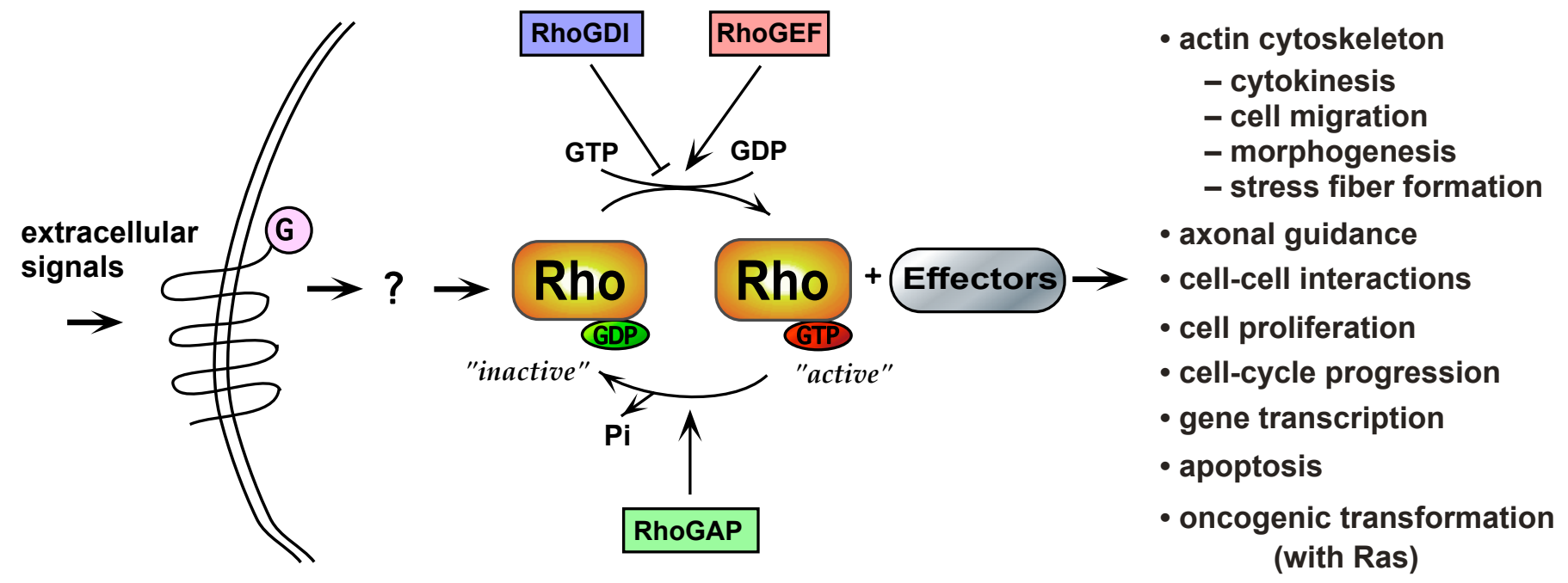


Figure 2.

(Johndrow, Magie \& Parkhurst)
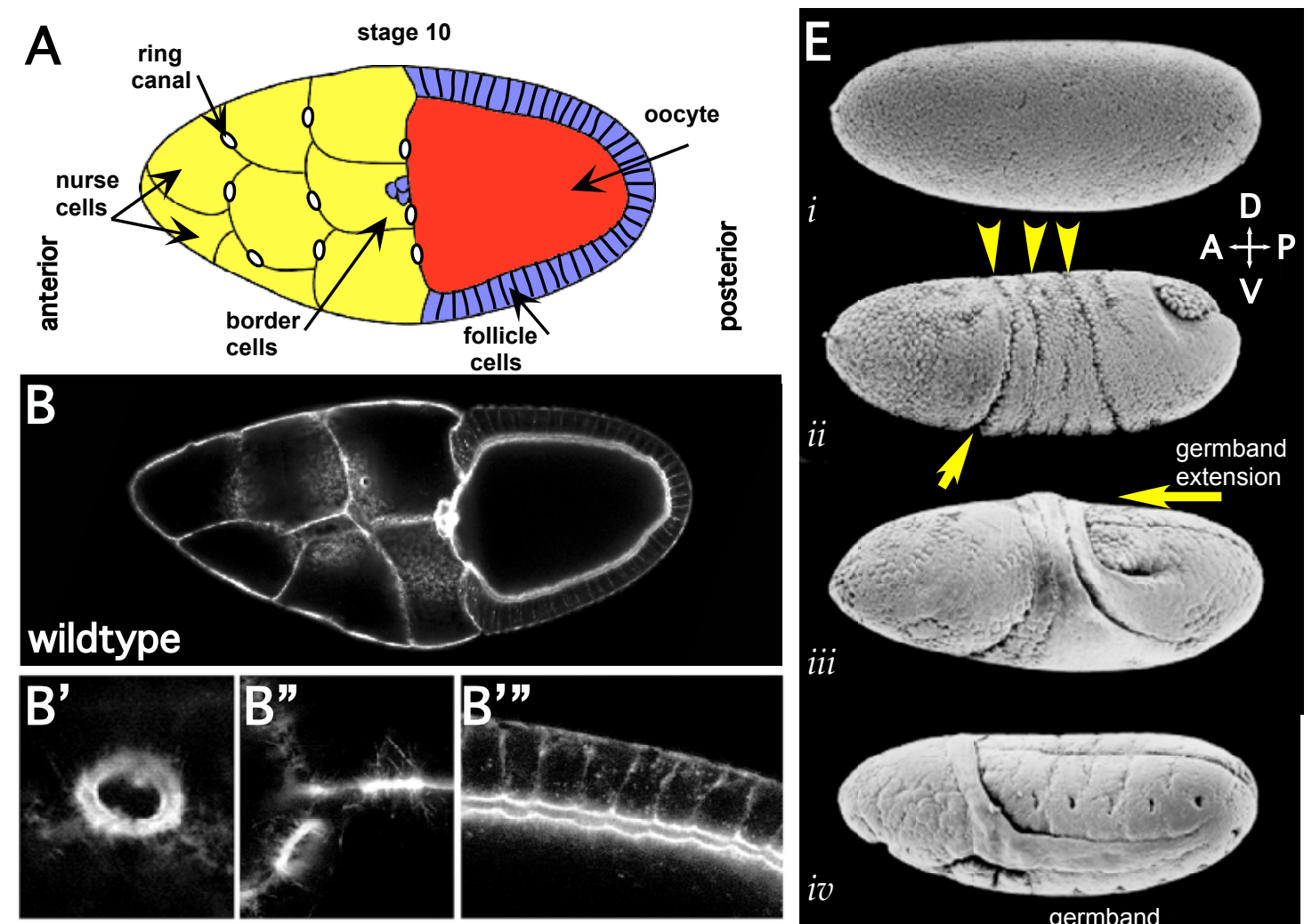

C

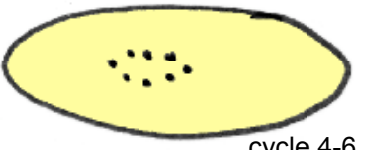

D
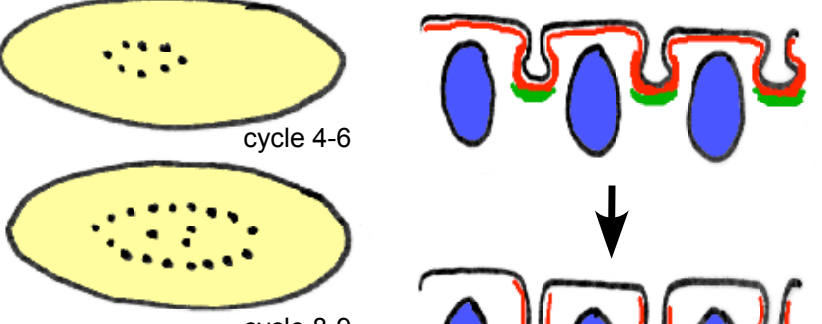

cycle 8-9
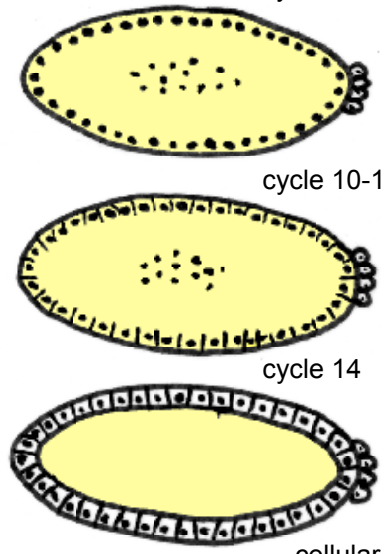

cellular
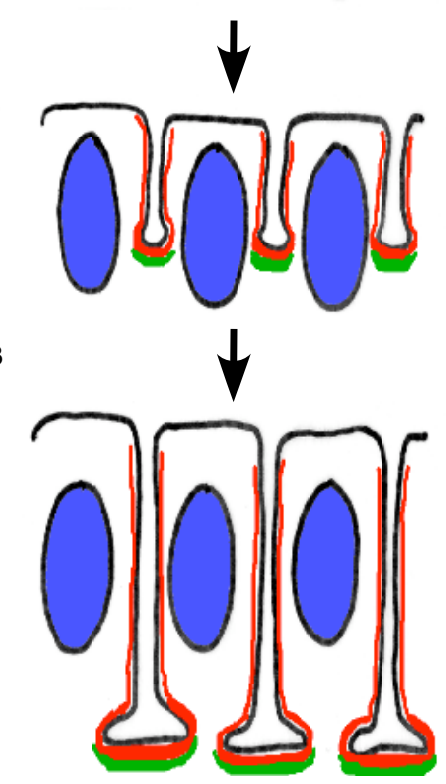

iii
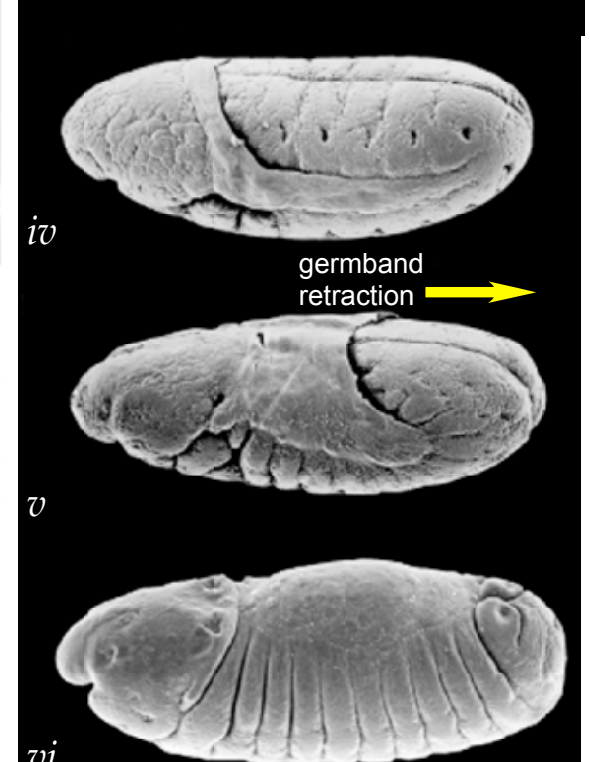

$v i$

dorsal closure

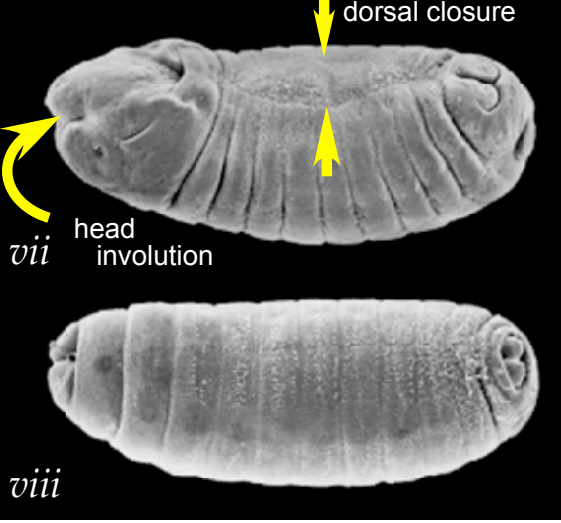


Figure 3.

(Johndrow, Magie \& Parkhurst)

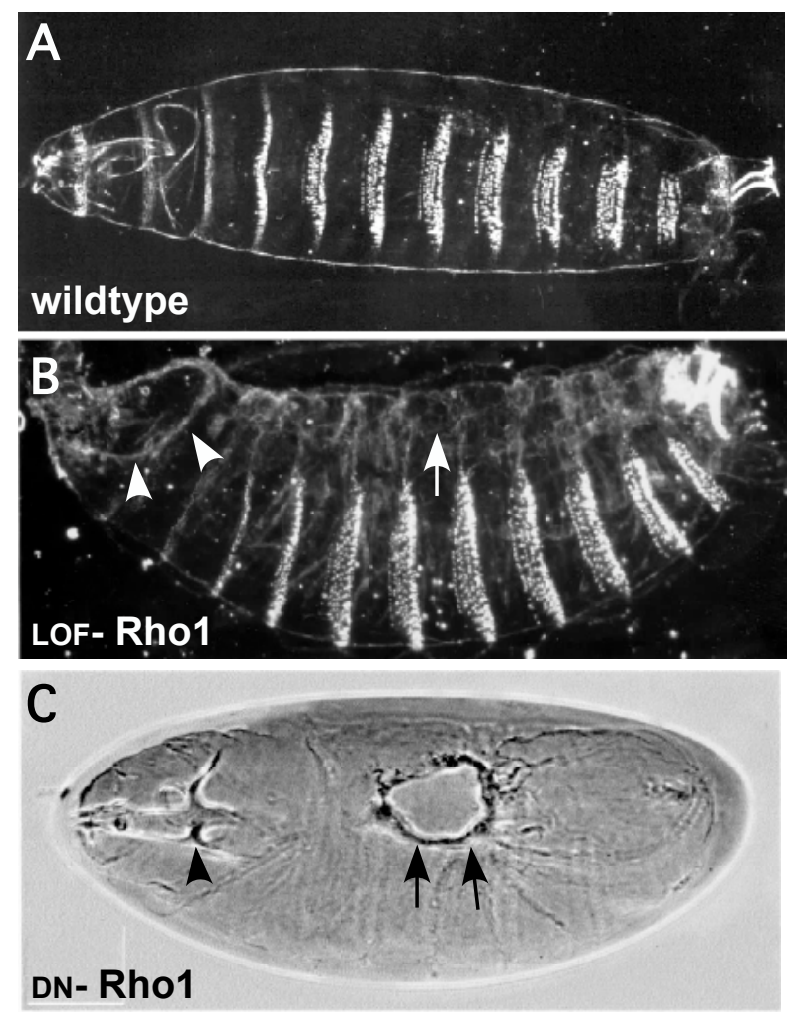


Figure 4.

(Johndrow, Magie \& Parkhurst)

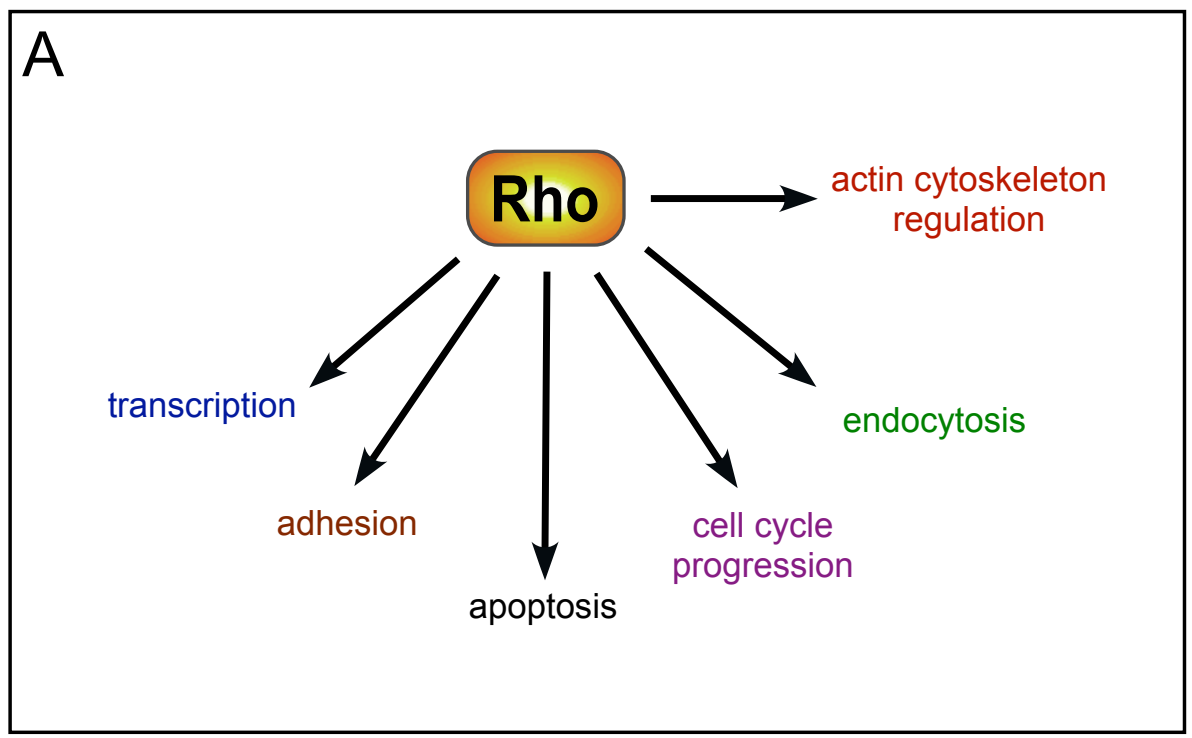

B
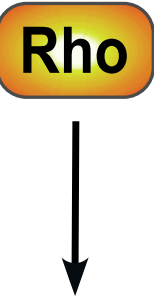

actin cytoskeleton

regulation

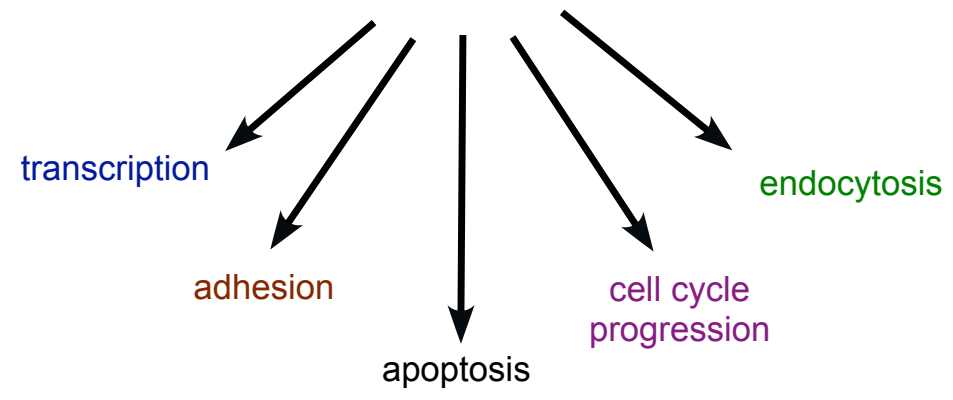

